\title{
25 Research Square \\ Synthesis, Antioxidant activity and Structure-Activity Relationship of gallic hydrazones Analogues
}

\author{
Zhengrong Wu \\ Lanzhou University

\section{Peng Chen} \\ Lanzhou University \\ Ying-Qian Liu ( 412725278@qq.com ) \\ Lanzhou University
}

\section{Research Article}

Keywords: gallic acid, hydrazone, antioxidant activity, Structure-Activity Relationship

Posted Date: October 20th, 2021

DOl: https://doi.org/10.21203/rs.3.rs-968882/v1

License: (1) This work is licensed under a Creative Commons Attribution 4.0 International License.

Read Full License 


\section{Abstract}

A series of gallic acid hydrazones were designed and synthesized as new potential anti-oxidant agents. Most of these compounds are potent antioxidants. The strongest compounds are 11 and $15\left(\mathrm{EC}_{50}: 6.42\right.$ $\left.\mu \mathrm{g} \cdot \mathrm{mL}^{-1}, 6.86 \mu \mathrm{g} \cdot \mathrm{mL}^{-1}, \mathrm{DPPH}\right)$ and $\left(\mathrm{EC}_{50}: 12.85 \mu \mathrm{g} \cdot \mathrm{mL}^{-1}, 12.49 \mu \mathrm{g} \cdot \mathrm{mL}^{-1} \mathrm{ABTS}\right)$, more potent than the positive control Trolox. Furthermore, the promising compounds 11 and 15 exhibited very low cytotoxic activity against HEK293 cell $\left(\mathrm{IC}_{50}>56.4 \mu \mathrm{M}\right)$. The SAR study revealed that the pattern of hydroxyl, methoxy and methyl substituents on the gallic hydrazones framework can increase the antioxidant properties of the prototype compounds. Moreover, the results also showed that the activity increased with the number of the groups and increased following hydroxyl > methoxy > methyl. Overall, the present study suggests that the designed compounds may serve as lead molecules for developing novel anti-oxidative agents in food industry.

\section{Introduction}

Free radicals and ROS play an important role in the pathogenesis of many diseases, the identification and synthesis of novel antioxidant to combat the harmful effects of free radicals and ROS attracting much attention for their versatile properties in pharmacology [1-4]. In particular, natural antioxidants, such as vitamin C, gallic acid, and polyphenols, are widely used to scavenge free radicals and to combat the harmful effects of ROS [5-10]. It is well known that donating group such as phenolic hydroxyl hydrazino and amino groups showed positive influence for the antioxidant activities, many efforts have been focussed on designing antioxidants containing phenolic hydroxyl groups [2, 11, 12]. Gallic acid which has three phenolic hydroxyl groups is a well-known natural antioxidant exhibits extensively biological activities, such as anti-oxidative, anti-tyrosinase, anti-inflammatory, anti-fungal, anti-cancer, and so on, which has versatile applications in medicine, food and pharmaceutical industries because of its unique physiochemical characteristics, non-toxicity, biodegradability, abundant availability, and low cost [13-17].

Recently, hydrazones were reported as potent antioxidants due to azomethine group increasing free radical scavenging ability have been extensively investigated $[5,7,18]$. Meanwhile, the $\mathrm{N}-\mathrm{H}$ proton of phenylhydrazones is necessary for the radical scavenging activity of this compound in Fig 1 [19].

Based on the findings and in continuation of our works on the field [20], the present work report here the synthesis and antioxidant properties of gallic acid hydrazones and their structure-activity relationships based on the presence, position and number of different substituents (hydroxyl, methoxy and methyl) on the phenyl ring.

\section{Results And Discussion}

\section{Synthesis}


The gallic acid esters were prepared from gallic acid in refluxing methanol and the presence of $\mathrm{H}_{2} \mathrm{SO}_{4}$ as catalyst. The reaction of esters with hydrazine hydrate in ethanol afforded hydrazides. Finally, condensation of hydrazide with different aromatic aldehydes in methanol produced the desired gallic acid hydrazones Analogues 1-14 in 68-85\% yield.

\section{Antioxidant activity}

The antioxidant activities of the newly prepared compounds were measured against DPPH and ABTS radicals assay respectively, according to the literatures [21, 22]. Trolox and gallic acid were also determined for comparison.

The results indicated that most the synthesized compounds possessed potent DPPH radical scavenging activity compared to the positive control Trolox. As shown in Table 1, most the synthesized compounds possessed potent DPPH radical scavenging activity compared to the positive control Trolox and gallic acid. The antioxidant activity of gallic acid hydrazones were enhanced in comparison with gallic acid, it could be concluded that $\mathrm{NH}$ group was important contributors to their DPPH radical scavenging activity. The derivatives 11 and 15, both containing six hydroxyl groups with $\mathrm{EC}_{50}$ value of: $6.42 \mu \mathrm{g} \cdot \mathrm{mL}^{-1}$ and 6.86 $\mu \mathrm{g} \cdot \mathrm{mL}^{-1}$ were the most efficient highlighting the importance of hydroxyl group. Compounds 8,9 and 12 although less active than compounds 11 and 15, also exhibit prominent DPPH radical scavenging activity, the results are in agreement with previous study that catechol group is the main contributor to the antioxidant capacity of phenolic compounds [23].

Additionally, introduction of methyl groups on the phenyl ring in the 2- and 3-positions increases the effects but the higher activity is obtained with 2- hydroxyl or 3- hydroxyl. The effect of hydroxy' s position is also studied, compounds 2, 4 and 7 differing in the position of the hydroxyl group, compounds 7 (4position) displayed better activities than compounds 2 (2-position) and 4 (3-position), the relative DPPH radical scavenging activity decreases in the following sequence: 4-position > 3-position > 2-position.

The same tendency was observed in the ABTS radical scavenging activity studies. As shown in Table 1. Most of synthesized compounds showed significant ABTS radical cation scavenging activity, the order of ABTS radical cation scavenging activity of the antioxidants was mostly in accordance with DPPH radical scavenging activity. The compounds (11 and 15) with $\mathrm{EC}_{50}$ value of $12.85 \mu \mathrm{g} \cdot \mathrm{mL}^{-1}$ and 12.49 $\mu \mathrm{g} \cdot \mathrm{mL}^{-1}$ were the most potent compounds too.

In view of the results, the number of active groups was highly related to the antioxidant activity of the compound, and generally more active groups resulted in higher activity. Additionally, electron donating substituent like methoxy can increase their antioxidant activity too and increased following hydroxyl > methoxy > methyl. Such phenomenon was consistent with previous study [24]. In summary, the hydrogendonating ability of phenoxyl radical influence the antioxidant activity of the prototype compounds. 
In order to check the safety profile of promising antioxidant, it was selected to test its cytotoxicity against human normal cell line (HEK293). The result showed that compounds 11 and 15 displayed much lower

cytotoxic activity for HEK293 $\left(\mathrm{IC}_{50}>56.4 \mu \mathrm{g} \cdot \mathrm{mL}^{-1}\right)$. The result revealed that the compounds 11 and 15 can display significant antioxidant activity at low concentrations without inducing cytotoxicity.

\section{Conclusions}

In conclusion, we have designed and synthesized a number of gallic acid hydrazones as new potential antioxidant. The in vitro antioxidant properties of the compounds in terms of reducing ability and radical scavenging activity were assessed by using ABTS and DPPH tests, respectively. Compound 11 and 15 which both have six hydroxyl groups were the most potent radical scavenger in the tsets. This finding might be attributed to the fact that the antioxidant activity was strongly dependent on the number of hydroxyl groups in the grafted phenolic moieties. The SAR study revealed that the pattern of hydroxyl, methoxy and methyl substituents on the gallic acid hydrazones framework can increase the antioxidant properties of the prototype compounds. Furthermore, the results also showed that the activity increased with the number of the groups and increased following hydroxyl > methoxy $>$ methyl.

Therefore, it could be concluded that this class of compounds had a good safety profile for their potential application in the food industry and proved that compound 11 and 15 might be potentially used as an antioxidant agents with a high potency and low toxicity in pharmaceutical, and food industries. Further studies on the relevant action mechanisms and structural modification of identified hits are on-going.

\section{Materials And Methods}

\section{Chemistry}

All reactions were performed with commercially available reagents and solvents without further purification. All reactions were monitored by thin-layer chromatography (TLC). ${ }^{1} \mathrm{H}$ and ${ }^{13} \mathrm{C}$ NMR spectra were recorded on Bruker AVANCE-III HD 400MHz or NEO 500MHz (Bruker Daltonics Inc., Germany) spectrometers using TMS as a reference. Mass spectra were recorded on a Bruker APEXII49e spectrometer (Bruker Daltonics Inc., Germany) with ESI source as ionization.

\section{General procedure for the synthesis of gallic hydrazones 1-17.}

The reaction route is outlined in Scheme 1. The intermediate compounds gallic hydrazide was prepared according to the reported methods [20]. Briefly, the gallic acid esters were prepared from gallic acid in refluxing methanol and the presence of $\mathrm{H}_{2} \mathrm{SO}_{4}$ as catalyst. The reaction of esters with hydrazine hydrate in ethanol afforded hydrazides. Finally, condensation of hydrazide with different aromatic aldehydes in methanol produced the desired gallic hydrazones analogues $1-17$ in $68-85 \%$ yield.

(E)-N'-benzylidene-3, 4, 5-trihydroxybenzohydrazide (1). white solid; yield: 85\%; ${ }^{1} \mathrm{H}$ NMR (400 MHz, DMSO)

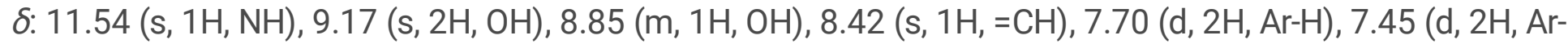


H), 6.93(s, 2H, Ar-H); ${ }^{13} \mathrm{C}$ NMR (100 MHz, DMSO) $\delta: 164.20,146.74,146.02,136.80,134.18,130.26$, $129.28,126.26,123.81,106.88$; ESI-MS: $m / z 273.10[\mathrm{M}+\mathrm{H}]^{+}$.

(E)-3, 4, 5-trihydroxy-N'-(4-hydroxybenzylidene) benzohydrazide (2). yellow solid; yield: 80\%; ${ }^{1} \mathrm{H}$ NMR (400 $\mathrm{MHz}, \mathrm{DMSO}) \delta: 11.53$ (s, 1H, NH), $9.16(\mathrm{~s}, 2 \mathrm{H}, \mathrm{OH}), 8.82(\mathrm{~s}, 2 \mathrm{H}, \mathrm{OH}), 8.42(\mathrm{~s}, 1 \mathrm{H},=\mathrm{CH}), 7.70(\mathrm{~m}, 2 \mathrm{H}, \mathrm{Ar}-\mathrm{H})$, 7.45 (m, 2H, Ar-H), 6.93(s, 2H, Ar-H); ${ }^{13} \mathrm{C}$ NMR (100 MHz, DMSO) $\delta: 163.88,147.64,146.02,138.00$, $135.00,130.70,129.27,128.07,124.22,121.89,117.82,107.66$; ESI-MS: $m / z 273.10[\mathrm{M}+\mathrm{H}]^{+}$; ESI-MS: $m / z$ $289.07[\mathrm{M}+\mathrm{H}]^{+}$.

(E)-3, 4, 5-trihydroxy-N'-(3-hydroxybenzylidene) benzohydrazide (3). yellow solid; yield: 76\%; ${ }^{1} \mathrm{H}$ NMR (400 MHz, DMSO) $\delta: 11.52(\mathrm{~s}, 1 \mathrm{H}, \mathrm{NH}), 10.24(\mathrm{~s}, 2 \mathrm{H}, \mathrm{OH}), 9.17(\mathrm{~s}, 1 \mathrm{H}, \mathrm{OH}), 8.73(\mathrm{~s}, 1 \mathrm{H},=\mathrm{CH}), 7.82(\mathrm{~m}, 2 \mathrm{H}, \mathrm{Ar}-\mathrm{H})$, 7.24-7.56 (m, 2H, Ar-H), 6.96(s, 2H, Ar-H), $2.56\left(\mathrm{~s}, 3 \mathrm{H}, \mathrm{CH}_{3}\right) ;{ }^{13} \mathrm{C}$ NMR (100 MHz, DMSO) $\delta: 163.46,145.31$, $140.59,137.42,137.11,134.36,133.03,129.50,126.60,125.73,123.84,107.50,19.41$; ESI-MS: $\mathrm{m} / \mathrm{z}$ $287.12[\mathrm{M}+\mathrm{H}]^{+}$.

(E)-3, 4, 5-trihydroxy-N'-(3-hydroxybenzylidene) benzohydrazide (4). white solid; yield: 80\%; ${ }^{1} \mathrm{H}$ NMR (400 $\mathrm{MHz}, \mathrm{DMSO}) \delta: 11.56(\mathrm{~s}, 1 \mathrm{H}, \mathrm{NH}), 10.21(\mathrm{~s}, 1 \mathrm{H}, \mathrm{OH}), 9.18(\mathrm{~s}, 2 \mathrm{H}, \mathrm{OH}), 8.86(\mathrm{~s}, 1 \mathrm{H}, \mathrm{OH}), 8.72(\mathrm{~s}, 1 \mathrm{H}$, $=\mathrm{CH}), 7.81$ (s, 2H, Ar-H), 7.23-1.55 (m, 2H, Ar-H), 7.01(s, 2H, Ar-H); ${ }^{13} \mathrm{C}$ NMR (100 MHz, DMSO) $\delta: 163.50$, 146.44, 144.41, 137.44, 135.09, 131.23, 129.27, 126.86, 123.40, 116.92, 107.66; ESI-MS: $\mathrm{m} / \mathrm{z} 273.10$ $[\mathrm{M}+\mathrm{H}]^{+}$; ESI-MS: $m / z 289.07[\mathrm{M}+\mathrm{H}]^{+}$.

(E)-3, 4, 5-trihydroxy-N'-(3-methylbenzylidene) benzohydrazide (5). yellow solid; yield: 70\%; ${ }^{1} \mathrm{H}$ NMR (400 MHz, DMSO) $\delta: 11.52$ (s, 1H, NH), $9.17(\mathrm{~s}, 2 \mathrm{H}, \mathrm{OH}), 8.83$ (s, 1H, OH), 8.38 (s, 1H, =CH), $7.82(\mathrm{~m}, 2 \mathrm{H}, \mathrm{Ar}-\mathrm{H})$, 7.22-7.53 (m, 4H, Ar-H), 6.94(s, 2H, Ar-H), $2.50\left(\mathrm{~s}, 3 \mathrm{H}, \mathrm{CH}_{3}\right) ;{ }^{13} \mathrm{C}$ NMR $(100 \mathrm{MHz}, \mathrm{DMSO}) \delta: 163.50,146.07$, $140.58,137.12,134.77,133.03,132.15,129.95,126.10,125.73,123.85,107.28,19.41$; ESI-MS: $m / z$ $287.12[\mathrm{M}+\mathrm{H}]^{+}$.

(E)-3, 4, 5-trihydroxy-N'-(3-methylbenzylidene) benzohydrazide (6). yellow solid; yield: 71\%; ${ }^{1} \mathrm{H}$ NMR (400 $\mathrm{MHz}, \mathrm{DMSO}) \delta: 11.57(\mathrm{~s}, 1 \mathrm{H}, \mathrm{NH}), 9.14(\mathrm{~s}, 2 \mathrm{H}, \mathrm{OH}), 8.82(\mathrm{~s}, 1 \mathrm{H}, \mathrm{OH}), 8.74(\mathrm{~s}, 1 \mathrm{H},=\mathrm{CH}), 7.37(\mathrm{~d}, 2 \mathrm{H}, \mathrm{Ar}-\mathrm{H})$, 6.94-7.06 (m, 4H, Ar-H), 3.81(s, 3H, $\left.\mathrm{CH}_{3}\right) ;{ }^{13} \mathrm{C}$ NMR (100 MHz, DMSO) $\delta: 163.08,154.36,152.66,145.99$, $137.42,123.70,117.75,113.90,109.64,107.28,56.75$; ESI-MS: $m / z 287.12[M+H]^{+}$.

(E)-3, 4, 5-trihydroxy-N'-(4-hydroxybenzylidene) benzohydrazide (7). white solid; yield: 68\%; ${ }^{1} \mathrm{H}$ NMR (400 MHz, DMSO) $\delta: 11.67(\mathrm{~s}, 1 \mathrm{H}, \mathrm{NH}), 11.63(\mathrm{~s}, 1 \mathrm{H}, \mathrm{OH}), 9.93(\mathrm{~s}, 1 \mathrm{H}, \mathrm{OH}), 9.18(\mathrm{~s}, 2 \mathrm{H}, 2 \mathrm{OH}), 8.45(\mathrm{~s}, 1 \mathrm{H}$, $=\mathrm{CH}), 7.24(\mathrm{~d}, 2 \mathrm{H}, \mathrm{Ar}-\mathrm{H}), 6.92(\mathrm{~s}, 2 \mathrm{H}, \mathrm{Ar}-\mathrm{H}), 6.34(\mathrm{~m}, 2 \mathrm{H}, \mathrm{Ar}-\mathrm{H}) ;{ }^{13} \mathrm{C}$ NMR $(100 \mathrm{MHz}, \mathrm{DMSO}) \delta:$ 163.07, 161.35, 148.97, 145.61, 137.54, 133.04, 123.34, 112.85, 108.03, 103.16; ESI-MS: $m / z 273.10$ [M+H] ${ }^{+}$; ESI-MS: $m / z$ $289.07[\mathrm{M}+\mathrm{H}]^{+}$.

(E)-N'-(2, 4-dihydroxybenzylidene)-3, 4, 5- trihydroxybenzohydrazide (8). white solid; yield: $75 \% ;{ }^{1} \mathrm{H}$ NMR (400 MHz, DMSO) $\delta: 11.40(\mathrm{~s}, 1 \mathrm{H}, \mathrm{NH}), 9.14(\mathrm{~s}, 2 \mathrm{H}, \mathrm{OH}), 8.95(\mathrm{~s}, 1 \mathrm{H}, \mathrm{OH}), 8.85(\mathrm{~s}, 1 \mathrm{H}, \mathrm{OH}), 8.29(\mathrm{~s}, 1 \mathrm{H}$, 
$=\mathrm{CH}), 6.91-6.95(\mathrm{~m}, 4 \mathrm{H}, \mathrm{Ar}-\mathrm{H}) ;{ }^{13} \mathrm{C}$ NMR $(100 \mathrm{MHz}, \mathrm{DMSO}) \delta: 163.38,148.61,147.64,146.00,138.11$, $137.27,135.37,133.56,125.73,124.08,107.28,104.96$; ESI-MS: $m / z 305.06[\mathrm{M}+\mathrm{H}]^{+}$.

(E)-N'-(2, 5-dihydroxybenzylidene)-3, 4, 5- trihydroxybenzohydrazide (9). white solid; yield: $69 \% ;{ }^{1} \mathrm{H} N M R$ (400 MHz, DMSO) 8:11.83 (s, 1H, NH), $11.41(\mathrm{~s}, 1 \mathrm{H}, \mathrm{OH}), 9.21(\mathrm{~s}, 1 \mathrm{H}, \mathrm{OH}), 9.12(\mathrm{~s}, 1 \mathrm{H}, \mathrm{OH}), 8.89(\mathrm{~s}, 1 \mathrm{H}$, $\mathrm{OH})$, , $9.12(\mathrm{~s}, 1 \mathrm{H},=\mathrm{CH}), 7.36(\mathrm{~d}, 2 \mathrm{H}, \mathrm{Ar}-\mathrm{H}), 7.05(\mathrm{~d}, 1 \mathrm{H}, \mathrm{Ar}-\mathrm{H}), 6.98(\mathrm{~m}, 1 \mathrm{H}, \mathrm{Ar}-\mathrm{H}), 6.94(\mathrm{~d}, 2 \mathrm{H}, \mathrm{Ar}-\mathrm{H}),{ }^{13} \mathrm{C} \mathrm{NMR}$ (100 MHz, DMSO) $\delta: 163.30,148.72,146.49,146.11,146.01,137.74,123.08,120.72,119.53,119.21$, 117.65, 107.66; ESI-MS: $m / z 305.06[\mathrm{M}+\mathrm{H}]^{+}$.

(E)-N'-(2, 5-dimethoxybenzylidene) -3, 4, 5- trihydroxybenzohydrazide (10). white solid; yield: 76\%; ${ }^{1} \mathrm{H}$ NMR (400 MHz, DMSO) $\delta: 11.57(\mathrm{~s}, 1 \mathrm{H}, \mathrm{NH}), 9.14(\mathrm{~s}, 2 \mathrm{H}, \mathrm{OH}), 8.82(\mathrm{~s}, 1 \mathrm{H}, \mathrm{OH}), 8.74(\mathrm{~s}, 1 \mathrm{H},=\mathrm{CH}), 7.36(\mathrm{~d}, 2 \mathrm{H}, \mathrm{Ar}-$ $\mathrm{H}), 7.05(\mathrm{~d}, 1 \mathrm{H}, \mathrm{Ar}-\mathrm{H}), 6.98(\mathrm{~m}, 1 \mathrm{H}, \mathrm{Ar}-\mathrm{H}), 6.94(\mathrm{~d}, 2 \mathrm{H}, \mathrm{Ar}-\mathrm{H}), 3.75\left(\mathrm{~s}, 3 \mathrm{H},-\mathrm{OCH}_{3}\right), 3.81\left(\mathrm{~s}, 3 \mathrm{H},-\mathrm{OCH}_{3}\right) ;{ }^{13} \mathrm{C}$ NMR (100 MHz, DMSO) $\delta: 163.38,153.73,152.63,141.77,137.41,123.77,118.13,114.92,113.88$, 110.71, 109.58, 107.28, 56.73, 55.91; ESI-MS: $m / z$ 301.11[M+H] $]^{+}$

(E)-3, 4, 5-trihydroxy-N'-(2, 3, 4-trihydroxybenzylidene) benzohydrazide (11). white solid; yield: $75 \% ;{ }^{1} \mathrm{H}$ NMR (400 MHz, DMSO) $\delta: 11.71(\mathrm{~s}, 1 \mathrm{H}, \mathrm{NH}), 11.65(\mathrm{~s}, 1 \mathrm{H}, \mathrm{OH}), 9.40(\mathrm{~s}, 1 \mathrm{H}, \mathrm{OH}), 9.19(\mathrm{~s}, 1 \mathrm{H}, \mathrm{OH}), 8.85(\mathrm{~s}$, $1 \mathrm{H}, \mathrm{OH}), 8.42(\mathrm{~s}, 1 \mathrm{H},=\mathrm{CH}), 6.93(\mathrm{~s}, 2 \mathrm{H}, \mathrm{Ar}-\mathrm{H}), 6.73(\mathrm{~d}, 1 \mathrm{H}, \mathrm{Ar}-\mathrm{H}), 6.38(\mathrm{~d}, 1 \mathrm{H}, \mathrm{Ar}-\mathrm{H}) ;{ }^{13} \mathrm{C} \mathrm{NMR}(100 \mathrm{MHz}$, DMSO) $\delta: 163.06,149.75,148.94,147.90,146.09,137.56,133.15,123.30,121.58,111.41,108.00,107.56$; ESI-MS: $m / z 321.05[\mathrm{M}+\mathrm{H}]^{+}$.

(E)-N'-(3, 4-dihydroxybenzylidene)-3, 4, 5- trihydroxybenzohydrazide (12). white solid; yield: $72 \% ;{ }^{1} \mathrm{H} \mathrm{NMR}$ (400 MHz, DMSO) $\delta: 11.71(\mathrm{~s}, 1 \mathrm{H}, \mathrm{NH}), 11.65(\mathrm{~s}, 1 \mathrm{H}, \mathrm{OH}), 9.40(\mathrm{~s}, 1 \mathrm{H}, \mathrm{OH}), 9.19(\mathrm{~s}, 2 \mathrm{H}, 2 \mathrm{OH}), 8.85(\mathrm{~s}, 1 \mathrm{H}$, $\mathrm{OH}), 8.42(\mathrm{~s}, 1 \mathrm{H},=\mathrm{CH}), 6.93(\mathrm{~s}, 2 \mathrm{H}, \mathrm{Ar}-\mathrm{H}), 6.73(\mathrm{~d}, 1 \mathrm{H}, \mathrm{Ar}-\mathrm{H}), 6.38(\mathrm{~d}, 1 \mathrm{H}, \mathrm{Ar}-\mathrm{H}),{ }^{13} \mathrm{C}$ NMR $(100 \mathrm{MHz}, \mathrm{DMSO}) \delta$ : 163.06, 149.75, 148.94, 147.90, 146.09, 137.55, 133.15, 123.30, 121.58, 111.41, 118.00, 107.56. ; ESI-MS: $\mathrm{m} / \mathrm{z} 305.06[\mathrm{M}+\mathrm{H}]^{+}$.

(E)-N'-(3, 4-dimethoxybenzylidene) -3, 4, 5- trihydroxybenzohydrazide (13). white solid; yield: 70\%; ${ }^{1} \mathrm{H}$ NMR (400 MHz, DMSO) $\delta: 11.57(\mathrm{~s}, 1 \mathrm{H}, \mathrm{NH}), 9.16(\mathrm{~s}, 2 \mathrm{H}, \mathrm{OH}), 8.83(\mathrm{~s}, 1 \mathrm{H}, \mathrm{OH}), 8.34(\mathrm{~s}, 1 \mathrm{H},=\mathrm{CH}), 7.02(\mathrm{~s}, 4 \mathrm{H}, \mathrm{Ar}-$ $\mathrm{H}), 6.98(\mathrm{~m}, 2 \mathrm{H}, \mathrm{Ar}-\mathrm{H}), 3.84\left(\mathrm{~s}, 6 \mathrm{H}, 2-\mathrm{OCH}_{3}\right) ;{ }^{13} \mathrm{C} \mathrm{NMR}(100 \mathrm{MHz}, \mathrm{DMSO}) \delta: 163.38,153.73,152.63,141.77$, $137.41,123.77,118.13,114.92,113.88,110.71,109.58,107.28,56.73,55.91$; ESI-MS: $m / z$ 301.11[M+H] ${ }^{+}$; ESI-MS: $m / z 333.13[\mathrm{M}+\mathrm{H}]^{+}$.

(E)-3, 4, 5-trihydroxy-N'-(3, 4, 5-trimethoxybenzylidene) benzohydrazide (14). white solid; yield: $78 \% ;{ }^{1} \mathrm{H}$ NMR (400 MHz, DMSO) $\delta: 11.54(\mathrm{~s}, 1 \mathrm{H}, \mathrm{NH}), 9.89(\mathrm{~s}, 1 \mathrm{H}, \mathrm{OH}), 9.17(\mathrm{~s}, 1 \mathrm{H}, \mathrm{OH}), 8.84(\mathrm{~s}, 1 \mathrm{H}, \mathrm{OH}), 8.34(\mathrm{~s}$, $1 \mathrm{H},=\mathrm{CH}), 7.26(\mathrm{~s}, 2 \mathrm{H}, \mathrm{Ar}-\mathrm{H}), 6.99(\mathrm{~s}, 2 \mathrm{H}, \mathrm{Ar}-\mathrm{H}), 3.85\left(\mathrm{~s}, 6 \mathrm{H}, 2-\mathrm{OCH}_{3}\right), 3.71\left(\mathrm{~s}, 3 \mathrm{H},-\mathrm{OCH}_{3}\right) ;{ }^{13} \mathrm{C}$ NMR $(100$ $\mathrm{MHz}$, DMSO) $\delta: 163.68,153.80,146.44,143.29,139.45,132.13,130.83,123.87,107.20,104.95,60.69$, 57.06; ESI-MS: $m / z 363.11[\mathrm{M}+\mathrm{H}]^{+}$. 
(E)-3, 4, 5-trihydroxy-N'-(3, 4, 5-trihydroxybenzylidene) benzohydrazide (15). Yellow solid; yield: $72 \% ;{ }^{1} \mathrm{H}$ NMR (400 MHz, DMSO) ${ }^{1} \mathrm{H}$ NMR (400 MHz, DMSO) $\delta: 11.32$ (s, 1H, NH), 9.33-9.09 (m, 4H, OH), 8.75 (s, $1 \mathrm{H}, \mathrm{OH}), 8.57(\mathrm{~s}, 1 \mathrm{H}, \mathrm{OH}), 8.08(\mathrm{~s}, 1 \mathrm{H},=\mathrm{CH}), 6.86(\mathrm{~s}, 2 \mathrm{H}, \mathrm{Ar}-\mathrm{H}), 6.60(\mathrm{~s}, 2 \mathrm{H}, \mathrm{Ar}-\mathrm{H}) ;{ }^{13} \mathrm{C} \mathrm{NMR}(100 \mathrm{MHz}$, DMSO) $\delta: 163.35,147.95,147.04,145.88,137.10,135.37,125.38,124.02,107.50,106.15$; ESI-MS: $\mathrm{m} / z$ $321.05[\mathrm{M}+\mathrm{H}]^{+}$.

(E)-3, 4, 5-trihydroxy-N'-(3, 4, 5-trimethoxybenzylidene) benzohydrazide (16). white solid; yield: $72 \% ;{ }^{1} \mathrm{H}$ NMR (400 MHz, DMSO) ${ }^{1} \mathrm{H}$ NMR (400 MHz, DMSO) $\delta: 11.35(\mathrm{~s}, 1 \mathrm{H}, \mathrm{NH}), 9.20(\mathrm{~s}, 2 \mathrm{H}, \mathrm{OH}), 8.79(\mathrm{~s}, 1 \mathrm{H}, \mathrm{OH})$, $8.25(\mathrm{~s}, 1 \mathrm{H},=\mathrm{CH}), 7.23(\mathrm{~s}, 1 \mathrm{H}, \mathrm{Ar}-\mathrm{H}), 6.90-7.00(\mathrm{~m}, 4 \mathrm{H}, \mathrm{Ar}-\mathrm{H}), 3.80\left(\mathrm{~s}, 3 \mathrm{H},-\mathrm{OCH}_{3}\right) ;{ }^{13} \mathrm{C} \mathrm{NMR}(100 \mathrm{MHz}$, DMSO) $\delta: 163.42,150.01,147.31,146.00,137.25,127.95,124.06,120.46,112.71,112.33,107.54,56.03$; ESI-MS: $m / z 319.10[\mathrm{M}+\mathrm{H}]^{+}$.

(E)-3, 4, 5-trihydroxy-N'-(4-hydroxy-3, 5-dimethoxybenzylidene) benzohydrazide (17). white solid; yield: 74\%; ${ }^{1} \mathrm{H}$ NMR (400 MHz, DMSO) $\delta: 11.41$ (s, $\left.1 \mathrm{H}, \mathrm{NH}\right), 9.14(\mathrm{~s}, 2 \mathrm{H}, \mathrm{OH}), 8.85(\mathrm{~s}, 2 \mathrm{H}, \mathrm{OH}), 8.29(\mathrm{~s}, 1 \mathrm{H},=\mathrm{CH})$, 6.93 (d, $m, 4 \mathrm{H}, \mathrm{Ar}-\mathrm{H}), 3.82\left(\mathrm{~s}, 6 \mathrm{H}, 2-\mathrm{OCH}_{3}\right) ;{ }^{13} \mathrm{C}$ NMR (100 MHz, DMSO) $\delta: 163.49,148.60,147.75,146.00$, $138.15,137.26,125.36,124.07,107.56,104.91,56.48$; ESI-MS: $m / z 349.09[\mathrm{M}+\mathrm{H}]^{+}$.

\section{Antioxidant activity assay}

\section{DPPH free radical-scavenging assay}

The DPPH radical-scavenging activities of test compounds were determined according to the reported method [14]. Briefly, the test compounds at various concentrations were added to $3 \mathrm{~mL}$ of DPPH solution ( $0.1 \mathrm{mM}$ in DMSO) and the reaction mixture was shaken vigorously. After incubation at room temperature for $10 \mathrm{~min}$, the absorbance of this solution was determined at $517 \mathrm{~nm}$ after 10, 30, and $60 \mathrm{~min}$, using a spectrophotometer. All of the assays were performed three times. The concentration of a certain compound necessary to decrease the initial DPPH concentration by $50 \%\left(\mathrm{EC}_{50} \mu \mathrm{g} / \mathrm{mL}\right)$ was determined by linear regression analysis of data obtained by plotting the scavenging rate $\%$ against the concentrations of that compound.

\section{ABTS radical-scavenging assay}

The ABTS radical-scavenging activities of test compounds were determined according to the reported method [22, 23]. ABTS radical cation was produced by reacting $7 \mathrm{mM}$ aqueous ABTS solution with 2.45 $\mathrm{mM}$ potassium persulphate and the mixture was allowed to stand in the dark at room temperature for $12-16 \mathrm{~h}$ before use. An aliquot $(0.1 \mathrm{~mL})$ of DMSO solution of different antioxidant concentrations was added to $3.9 \mathrm{~mL}$ of the ABTS solution. Absorbance at $734 \mathrm{~nm}$ was recorded at different time intervals on a UV-vis spectrophotometer.

\section{Cytotoxicity assay}


Considering that compounds 11 and 15 were the most potent antioxidant agents, they were selected to investigate the cytotoxicity against human normal cell line (HEK293) according to the reported method $[24,25]$. Cells were seeded in 96-well plates and then treated with different concentrations of compounds 11 and 15 , they were dissolved in DMSO as a $100 \mu \mathrm{M}$ stock solution and then diluted to the different concentrations. Two independent experiments in triplicate were done for determination of cell viability inhibition for the compound. The $\mathrm{IC}_{50}$ values were calculated from concentration-response curves and expressed as means $\pm \mathrm{SD}$.

\section{Conclusion}

We have designed and synthesized a number of gallic acid hydrazones as new potential antioxidant. The in vitro antioxidant properties of the compounds in terms of reducing ability and radical scavenging activity were assessed by using ABTS and DPPH tests, respectively. Compound 11 and 15 which both have six hydroxyl groups were the most potent radical scavenger in the tsets.

This finding might be attributed to the fact that the antioxidant activity was strongly dependent on the number of hydroxyl groups in the grafted phenolic moieties. Furthermore, cytotoxicity assay showed that they had lower cytotoxic activity for HEK293.

Therefore, it could be concluded that this class of compounds had a good safety profile for their potential application in the food industry and proved that compound 11 and 15 might be potentially used as novel antioxidant agents with a high potency and low toxicity.

The SAR study revealed that the pattern of hydroxyl, methoxy and methyl substituents on the gallic acid hydrazones framework can increase the antioxidant properties of the prototype compounds. Furthermore, the results also showed that the activity increased with the number of the groups and increased following hydroxyl > methoxy > methyl. Overall, the present study suggests that the designed compounds may serve as lead molecules for developing novel anti-oxidative agents in pharmaceutical, cosmetic, and food industries.

Table 1 : Antioxidant activities (DPPH rdical savenging ativity and ABTS scavenging activity) of compounds 1-17. 


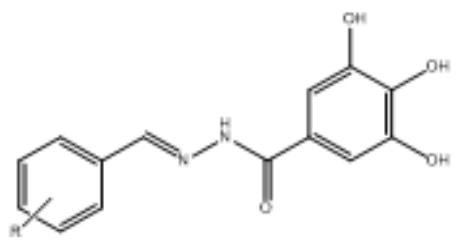

\begin{tabular}{|c|c|c|c|}
\hline \multirow{2}{*}{ Compound } & \multirow{2}{*}{$\mathrm{R}$} & \multicolumn{2}{|c|}{ Antioxidant activity } \\
\hline & & $\mathrm{DPPH} \quad \mathrm{EC}_{50}\left(\mu \mathrm{g} \cdot \mathrm{mL}^{-1}\right)$ & ABTS $\quad \mathrm{EC}_{50}\left(\mu \mathrm{g} \cdot \mathrm{mL}^{-1}\right)$ \\
\hline 1 & $\mathrm{H}$ & $46.35 \pm 7.35$ & $56.40 \pm 8.81$ \\
\hline 2 & $2-\mathrm{OH}$ & $30.04 \pm 5.02$ & $32.27 \pm 6.19$ \\
\hline 3 & $2-\mathrm{CH}_{3}$ & $45.20 \pm 6.70$ & $52.70 \pm 5.84$ \\
\hline 4 & $3-\mathrm{OH}$ & $26.54 \pm 4.17$ & $31.48 \pm 5.12$ \\
\hline 5 & $3-\mathrm{CH}_{3}$ & $35.85 \pm 6.08$ & $50.63 \pm 7.15$ \\
\hline 6 & $3-\mathrm{OCH}_{3}$ & $30.14 . \pm 4.79$ & $46.25 \pm 6.44$ \\
\hline 7 & $4-\mathrm{OH}$ & $25.08 \pm 4.15$ & $32.59 \pm 5.08$ \\
\hline 8 & $2,4-(\mathrm{OH})_{2}$ & $19.39 \pm 4.05$ & $23.16 \pm 2.20$ \\
\hline 9 & $2,5-(\mathrm{OH})_{2}$ & $15.71 \pm 3.69$ & $24.35 \pm 3.41$ \\
\hline 10 & $2,5-\left(\mathrm{CH}_{3}\right)_{2}$ & $38.28 \pm 5.35$ & $43.39 \pm 4.13$ \\
\hline 11 & $2,3,4-(\mathrm{OH})_{3}$ & $6.42 \pm 0.57$ & $12.85 \pm 2.15$ \\
\hline 12 & $3,4-(\mathrm{OH})_{2}$ & $20.73 \pm 4.08$ & $19.24 \pm 4.45$ \\
\hline 13 & $3,4-\left(\mathrm{OCH}_{3}\right)_{2}$ & $34.42 \pm 5.17$ & $40.62 \pm 3.58$ \\
\hline 14 & $3,4,5-\left(\mathrm{OCH}_{3}\right)_{3}$ & $32.53 \pm 6.45$ & $36.87 \pm 2.83$ \\
\hline 15 & $3,4,5-(\mathrm{OH})_{3}$ & $6.86 \pm 1.95$ & $12.49 \pm 3.02$ \\
\hline 16 & $3-\mathrm{OH}-4-\mathrm{OCH}_{3}$ & $30.42 \pm 3.52$ & $32.73 \pm 2.14$ \\
\hline 17 & $4-\mathrm{OH}-3,5-\left(\mathrm{OCH}_{3}\right)_{2}-$ & $26.43 \pm 2.75$ & $29.85 \pm 2.16$ \\
\hline gallic acid & - & $51.50 \pm 8.39$ & $60.15 \pm 9.37$ \\
\hline Trolox & - & $14.62 \pm 1.40$ & $22.08 \pm 4.63$ \\
\hline
\end{tabular}

Values are the mean \pm SD of three replicates.

\section{Declarations}

\section{Acknowledgements}

This work was supported financially by the National Natural Science Foundation of China (21877056 and 21672092) and the National Key Research and Development Program of China (2017YFD0201404).

\section{Compliance with ethics requirements}

Authors have no financial relationship with the organization that sponsored the research.

CRediT authorship contribution statement:

Zheng-Rong Wu: Software, Data curation, Writing-original draft. Chen Peng: Supervision. Ying-Qian Liu: Funding acquisition, Writing - review. Supervision. 
The authors declare that they have no known competing financial interests or personal relationships that could have appeared to influence the work reported in this paper.

\section{References}

1. Zhang Y, Zou B Q, Chen Zh F, Pan Y M, Wang H S, et al. Synthesis and antioxidant activities of novel 4-Schiff base-7-benzyloxy-coumarin derivatives. Bioorg Med Chem Lett. 2011; 21: 6811-6815.

2. Bandgar B P, Chavan H V, Adsul L K, Thakare V N, Shringare S N, Shaikh R, et al. Design, synthesis, characterization and biological evaluation of novel pyrazole integrated benzophenones. Bioorg Med Chem Lett. 2013; 23: 912-916.

3. Belkheiri N, Bouguerne B, Bedos-Belval F, Duran H, Bernis C, Salvayre R, et al. Synthesis and antioxidant activity evaluation of a syringic hydrazones family. Eur J Med Chem. 2010; 45: 30193026.

1. Sivakumar P M, rabhakar P K, Doble M. Synthesis, antioxidant evaluation, and quantitative structure-activity relationship studies of chalcones. Med Chem Res. 2010; 20(4): 482-492.

2. Carradori S, Ortuso F, Petzer A, Bagetta D, De Monte, et al. Design, synthesis and biochemical evaluation of novel multi-target inhibitors as potential anti-Parkinson agents. Eur $\mathrm{J}$ Med Chem. 2018; 143: 1543-1552.

3. Carcelli M, Rogolino D, Bartoli J, Pala N, Compari C, Ronda N, et al. Hydroxyphenyl thiosemicarbazones as inhibitors of mushroom tyrosinase and antibrowning agents. Food Chem. 2020; 303:125310.

4. Emami S, Esmaili Z, Dehghan G, Bahmani M, Hashemi S M, Mirzaei M S. et al. Acetophenone benzoylhydrazones as antioxidant agents: Synthesis, in vitro evaluation and structure-activity relationship studies. Food Chem. 2018; 92-299.

5. Hazer B, Ashby R D. Synthesis of a novel tannic acid-functionalized polypropylene as antioxidant active-packaging materials. Food Chem. 2021; 344, 128644.

6. Peng Z Y, Wang G C, Zeng Q H, Li Y F, Wu Y, Liu H Q, er al. Synthesis, antioxidant and anti-tyrosinase activity of 1, 2, 4-triazole hydrazones as antibrowning agents. Food Chem. 2021: 128265.

7. Rakesh K P, Manukumar H M, Gowda D C. Schiff's bases of quinazolinone derivatives: Synthesis and SAR studies of a novel series of potential anti-inflammatory and antioxidants. Bioorg Med Chem Lett. 2015, 25(5): 1072-1077.

8. Bendary E, Francis R R, Ali H M G, Sarwat M I, El-Hady S. Antioxidant and structure-activity relationships (SARs) of some phenolic and anilines compounds. AOAS. 2013, 58(2): 173-181.

9. Lee D S, Woo J Y, Ahn C B, Je J Y. Chitosan-hydroxycinnamic acid conjugates: Preparation, antioxidant and antimicrobial activity. Food Chem. 2014, 148: 97-104.

10. Yilmaz Y, Toledo R T. Major Flavonoids in Grape Seeds and Skins: Antioxidant Capacity of Catechin, Epicatechin, and Gallic Acid. J Agric Food Chem. 2004, 52(2): 255-260. 
11. Chen H M, Wu Y C, Chia Y C, Chang F R Yuan S S. Gallic acid, a major component of Toona sinensis leaf extracts, contains a ROS-mediated anti-cancer activity in human prostate cancer cells. Cancer Lett. 2009, 286(2):161-171.

12. Hong Z, Tang P, Liu B, Ran C, Wu H. Ferroptosis-related Genes for Overall Survival Prediction in Patients with Colorectal Cancer can be Inhibited by Gallic acid. Int J Bio Sci. 2021, 17(4): 942-956.

13. Barontini M , Silvestri I P, Nardi V, Crisante F囚 Pepe G『L Pari囚et al. Synthesis and biological evaluation of gallic acid analogs. Med Chem Res. 2012, 22(2): 674-680.

14. Shah S T, Yehye W A, Saad O, Simarani K, Chowdhury Z Z, Alhadi A A, et al. Surface Functionalization of Iron Oxide Nanoparticles with gallic acid as potential antioxidant and antimicrobial agents. Nanomaterials. 2017, 10, 3-17.

15. Bendary E, Francis R R, Ali H M G, Sarwat M I, El-Hady S. Antioxidant and structure-activity relationships (SARs) of some phenolic and anilines compounds. AOAS. 2013; 58(2):173-181.

16. Zhang M, Dai Z C, Qian S S, Liu J Y, Xiao Y, et al. Design, Synthesis, Antifungal, and Antioxidant Activities of (E)-6-((2-Phenylhydrazono) methyl) quinoxaline Derivatives. J Agric Food Chem. 2014; 62: 9637-9643.

17. Wu Z R, Zheng L F, Li Y, Su F, Yue X X, Tang W, et al. Synthesis and structure-activity relationships and effects of phenylpropanoid amides of octopamine and dopamine on tyrosinase inhibition and antioxidation. Food Chem. 2012, 134: 1128-1133.

18. Pan Y, He C, Wang H, Ji X \Kai W囚Liu P. Antioxidant activity of microwave-assisted extract of Buddleia officinalis and its major active component. Food Chem. 2010, 121(2): 497-502.

19. Fu Y Q, Zhang Y, Hu H Y, Chen Y, Wang R, Li D, et al. Design and straightforward synthesis of novel galloyl phytosterols with excellent antioxidant activity. Food Chem. 2014, 163: 171-177.

20. Olajide T M, Liu T, Liu H A, Weng, X. Antioxidant properties of two novel lipophilic derivatives of hydroxytyrosol. Food Chem. 2020, 315:126197.

21. Al-Madhagi W M, Hashim N M, Othman R, Ali N. Phytochemical screening, cytotoxic and antimicrobial activities of Limonium socotranum and Peperomia blanda extracts. Trop Biomed. 2019, 1(36):11-21.

22. Wang G, Liu W, Gong Z, Huang Y, Li Y, Peng Z. Synthesis, biological evaluation, and molecular modelling of new naphthalene-chalcone derivatives as potential anticancer agents on MCF-7 breast cancer cells by targeting tubulin colchicine binding site. J Enzym Inhib Med Ch. 2020, 35(1): 139-144.

\section{Figures}


<smiles>Cc1cccc(/C=N/NC(=O)c2ccccc2)c1</smiles>

Phenylhydrazones containing $\mathrm{OH}$ and $\mathrm{NH}$ groups

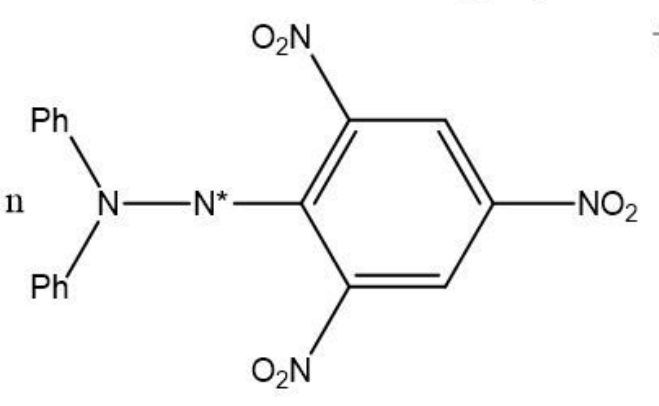

DPPH Radical<smiles>Cc1cccc(/C=N/NC(=O)c2ccccc2)c1</smiles>

Phenylhydrazones Radical

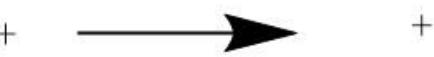

$\mathrm{n}$

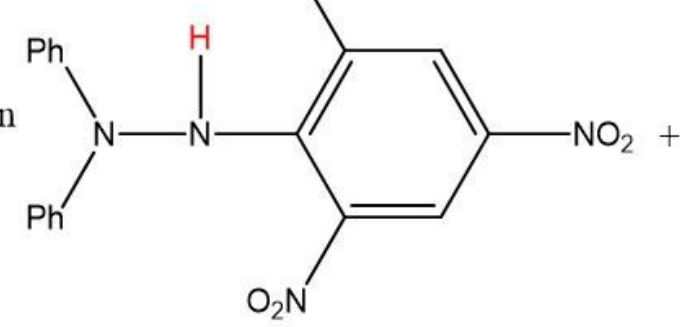

$\mathrm{DPPH}$ in reduced form

\section{Figure 1}

Reaction pattern of hydroxyl phenylhydrazones with DPPH radica proposed by predecessors with modification [19], in which the $\mathrm{NH}$ and $\mathrm{OH}$ are necessary for the radical scavenging activity.

\section{Supplementary Files}

This is a list of supplementary files associated with this preprint. Click to download.

- graphicsabstract.jpg

- scheme1.jpg

- Supplementarymaterial.doc 